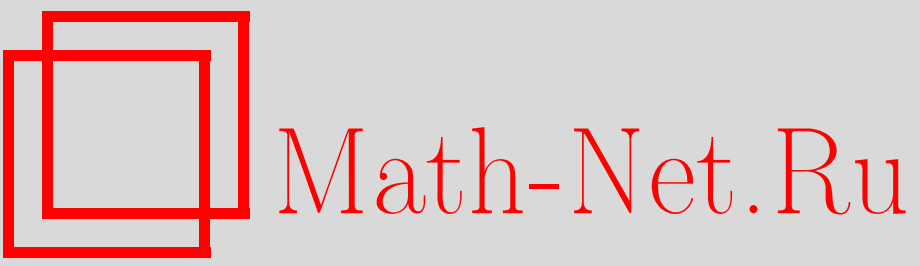

Д. К. Демской, Об одном классе систем лиувиллевского типа, ТМФ, 2004, том 141, номер 2, 208-227

DOI: https://doi.org/10.4213/tmf121

Использование Общероссийского математического портала Math-Net.Ru подразумевает, что вы прочитали и согласны с пользовательским соглашением

http://www . mathnet.ru/rus/agreement

Параметры загрузки:

IP : 44.207 .124 .84

26 апреля 2023 г., 06:36:31 
ТЕОРЕТИЧЕСКАЯ

И МАТЕМАТИЧЕСКАЯ

ФИЗИКА

Том 141, № 2

ноябрь, 2004

(C) 2004 г.

Д.К. Демской*

\section{ОБ ОДНОМ КЛАССЕ СИСТЕМ ЛИУВИЛЛЕВСКОГО ТИПА}

Доказана лиувиллевость одного класса систем с лагранжианом вида $L=$ $\left[g_{i j}(u) u_{x}^{i} u_{t}^{j}\right] / 2+f(u)$. Построены новые интегрируемые гамильтоновы системы, связанные дифференциальными подстановками типа преобразования Миуры с симметриями рассматриваемых гиперболических систем. Для одной из полученных систем построен рекурсионный оператор второго порядка.

Ключевые слова: системы лиувиллевского типа, высшие псевдоконстанты, рекурсионный оператор.

\section{1. ВВЕДЕНИЕ}

Открытие метода обратной задачи рассеяния позволило проинтегрировать ряд уравнений, играюших важную роль в описании нелинейных волн самой различной природы. K этим уравнениям относятся, в частности, уравнение Кортвега-де Фриза, нелинейное уравнение Шредингера, уравнение sh-Гордон и др. Как оказалось, эти уравнения обладают широкими классами точных решений, среди которых наиболее известны солитонные. Этот факт возродил интерес исследователей к методам точного интегрирования как таковым, а также позволил переосмыслить ряд известных еще с конца XIX в. фактов, связанных с приложениями нелинейных гиперболических уравнений в дифференциальной геометрии. Систематическое исследование нелинейных гиперболических уравнений с новых позиций было начато в работах [1], где были исследованы уравнения вида $u_{x t}=F(u)$. Было показано, что интегрируемыми над полем комплексных чисел являются только три уравнения:

$$
\begin{aligned}
& u_{x t}=a e^{u} \\
& u_{x t}=a e^{u}+b e^{-2 u} \\
& u_{x t}=a e^{u}+b e^{-u}
\end{aligned}
$$

где $a, b=$ const. Это хорошо известные уравнения Лиувилля, Цицейки и sh-Гордон. Нужно отметить, что все они были известны еще в начале ХХ в. Приведенные урав-

\footnotetext{
* Орловский государственный университет, Орел, Россия. E-mail: demskoy@iname.com
} 
нения представляют два различных класса интегрируемых уравнений: уравнения Цицейки и sh-Гордон являются интегрируемыми методом обратной задачи рассеяния, а для уравнения Лиувилля известна формула общего решения

$$
u=\ln \left(2 \frac{X^{\prime} T^{\prime}}{a(X+T)^{2}}\right), \quad X=X(x), \quad T=T(t) .
$$

Несмотря на различие этих классов, между ними существует тесная связь. Уравнение Лиувилля является вырождением уравнений sh-Гордон и Цищейки, вследствие чего высшие симметрии этих уравнений имеют одинаковую структуру:

$$
u_{\tau}=M G_{k}\left(\omega, \omega_{x}, \ldots\right),
$$

где

$$
M=D+u_{x}, \quad \omega=u_{x x}-\frac{1}{2} u_{x}^{2} .
$$

Для уравнений (1б), (1в) $G_{k}$ - дискретные последовательности функций; например, для (1б) эта последовательность имеет вид

$$
G_{k+1}=\left(D^{2}+2 \omega-D^{-1} \omega_{x}\right) G_{k}, \quad G_{0}=1 .
$$

В случае уравнения Лиувилля $G$ - произвольная функция своих аргументов, а функция $\omega$ удовлетворяет характеристическому уравнению

$$
D_{t} \omega=0,
$$

где $D_{t}$ - оператор полного дифференцирования по переменной $t$. Решения уравнения (3) называются $t$-псевдоконстантами ( $t$-интегралами). Понятно, что далеко не каждое гиперболическое уравнение обладает псевдоконстантами, однако, если это свойство выполняется, то уравнение может быть явно проинтегрировано (см., например, [2]-[4]). Поэтому уравнение Лиувилля, а также другие уравнения, обладаюшие псевдоконстантами, представляют особый интерес. В работах [2], [5], [6] приводятся результаты классификации уравнений, обладающих псевдоконстантами не выше второго порядка, а в работе [7] показано, что сушествуют уравнения, имеюшие псевдоконстанты порядка больше 2.

Обобшением уравнения Лиувилля для случая систем уравнений являются открытые двумеризованные цепочки Тоды [3], [8], которые можно записать в виде

$$
u_{x t}^{i}=\exp \left(A_{j}^{i} u^{j}\right), \quad i, j=1, \ldots, n .
$$

Здесь $A_{j}^{i}$ - элементы матрицы Картана простой алгебры Ли; по повторяющимся индексам подразумевается суммирование. Причем общим признаком как в случае скалярных уравнений, так и в случае систем является наличие псевдоконстант. Однако в последнем случае просто их наличие оказывается слишком слабым признаком интегрируемости. Например, для любой симметризуемой матрицы $A_{j}^{i}$ :

$$
\alpha_{i j}=\Lambda_{i s} A_{j}^{s}, \quad \alpha_{i j}=\alpha_{j i}, \quad \Lambda=\operatorname{diag}\left(a_{1}, \ldots, a_{n}\right),
$$

соответствующая система (4) допускает псевдоконстанту

$$
\rho=a_{i} u_{x x}^{i}-\frac{1}{2} \alpha_{i j} u_{x}^{j} u_{x}^{i}
$$

Поэтому требуется существование полного набора псевдоконстант по каждой характеристике. Точное определение выглядит следуюшим образом [9]. 
ОПРЕДЕЛЕниЕ 1. Система гиперболических уравнений

$$
u_{x t}^{i}=F^{i}\left(\mathbf{u}, \mathbf{u}_{x}, \mathbf{u}_{t}\right), \quad i=1, \ldots, n,
$$

называется системой лиувиллевского типа, если существуют $t$-псевдоконстанты и $x$ псевдоконстанты $\omega^{i}, \widetilde{\omega}^{i}, i=1, \ldots, n$, порядка соответственно $s$ и $p$, удовлетворяюшие условиям

$$
\operatorname{det}\left(\frac{\partial \omega^{i}}{\partial u_{s}^{j}}\right) \neq 0, \quad \operatorname{det}\left(\frac{\partial \widetilde{\omega}^{i}}{\partial \tilde{u}_{p}^{j}}\right) \neq 0 .
$$

Для того чтобы получить полный набор псевдоконстант для заданной системы, можно использовать несколько различных способов. Первый подход состоит в том, чтобы решить характеристическое уравнение (3), зафиксировав предварительно порядок псевдоконстанты. Этот способ проблематичен по двум причинам. Первая из них заключается в том, что порядок псевдоконстанты заранее не известен. Вторая проблема состоит в том, что решение получаюшихся в этом случае дифференциальных уравнений может оказаться технически непростой задачей, особенно в случае $\operatorname{ord}(\boldsymbol{\omega})>2$.

Иногда полезным оказывается то наблюдение, что уравнения лиувиллевского типа возникают как вырожденный случай уравнений, интегрируемых методом обратной задачи рассеяния. При этом плотности законов сохранения вторых являются одновременно псевдоконстантами для первых. Точнее, сохраняющиеся плотности с точностью до полных производных являются функциями псевдоконстант и их полных производных. Это означает, что можно использовать рекуррентные соотношения для законов сохранения, чтобы получить полные наборы псевдоконстант.

Еше один способ вычисления псевдоконстант опирается на тот хорошо известный факт (см., например, [2], [10]), что оператор полной производной в силу симметрии переводит псевдоконстанты в псевдоконстанты; следовательно, имея одну псевдоконстанту и невырож денную (определение см. ниже) симметрию, мы можем построить полный набор псевдоконстант. Этот факт приобретает прикладное значение именно в случае систем и, по-видимому, раньше для вычисления псевдоконстант не применялся. В качестве простого примера, иллюстрирующего эту идею, мы рассмотрим двухкомпонентную цепочку Тоды серии $A$ :

$$
u_{x t}=e^{2 u-v}, \quad v_{x t}=e^{-u+2 v} .
$$

Легко найти, что система (7) допускает полиномиальную симметрию второго порядка

$$
\begin{aligned}
& u_{\tau}=u_{x x}-2 v_{x x}-2 u_{x} v_{x}-u_{x}^{2}+2 v_{x}^{2}, \\
& v_{\tau}=-v_{x x}+2 u_{x x}+2 u_{x} v_{x}-2 u_{x}^{2}+v_{x}^{2} .
\end{aligned}
$$

Первая псевдоконстанта имеет вид

$$
\rho=u_{x x}+v_{x x}-u_{x}^{2}+u_{x} v_{x}-v_{x}^{2} .
$$


Чтобы получить вторую независимую псевдоконстанту, положим $\phi=D_{\tau} \rho$, где $D_{\tau}-$ оператор эволюционного дифференцирования в силу системы (8). Легко видеть, что $\operatorname{ord} \phi=4$, и, кроме того, $\phi \in \operatorname{Im} D$. Интегрируя $\phi$ по $x$, получим

$$
\hat{\theta}=D^{-1} \phi=3 u_{x x x}-3 v_{x x x}-6 u_{x x} u_{x}-3 u_{x x} v_{x}+3 v_{x x} u_{x}+6 v_{x x} v_{x}+6 u_{x}^{2} v_{x}-6 v_{x}^{2} u_{x} .
$$

Для упрощения полученной псевдоконстанты положим

$$
\theta=-\frac{1}{6} \hat{\theta}+\frac{1}{2} \rho_{x}=v_{x x x}+u_{x x} v_{x}-2 v_{x x} v_{x}-u_{x}^{2} v_{x}+v_{x}^{2} u_{x}
$$

Таким образом, справедливо соотношение

$$
\rho_{\tau}=3 \rho_{x x}-6 \theta_{x}
$$

Кроме того, можно сравнительно легко получить эволюционную систему, связанную дифференциальной подстановкой $(\rho, \theta)$ с системой (8). Для этого мы действуем оператором $D_{\tau}$ на $\theta$ и записьваем полученньй результат в терминах $\rho, \theta, \rho_{x}, \theta_{x}, \ldots$ Второе уравнение искомой системы будет иметь вид

$$
\theta_{\tau}=2 \rho_{x x x}-3 \theta_{x x}+\left(\rho^{2}\right)_{x}
$$

Система (9), (10) является одной из форм записи уравнения Буссинеска. В заключение этого примера отметим, что высшие симметрии системы (7) могут быть представлены в форме (2). Теорема 3 настояшей статьи дает способ отыскания оператора $M$, опирающийся на гамильтонову формулировку соответствующей гиперболической системы.

Рассматриваемые в настоящей работе системы принадлежат к классу лагранжевых релятивистски-инвариантных двумерных систем следующего вида:

$$
L=\frac{1}{2}\left(u_{t} u_{x}+\psi\left(v_{t} w_{x}+v_{x} w_{t}\right)\right)+f(u, v, w)
$$

где $\psi=(v w+c)^{-1}$. Лагранжиану (11) соответствуют полевые уравнения

$$
u_{t x}=\frac{\partial f}{\partial u}, \quad v_{t x}-\psi w v_{x} v_{t}=\psi^{-1} \frac{\partial f}{\partial w}, \quad w_{t x}-\psi v w_{t} w_{x}=\psi^{-1} \frac{\partial f}{\partial v} .
$$

В работе [11] показано, что существует только девять систем вида (12), допускаюших высшие полиномиальные симметрии не выше пятого порядка, причем соответствуюшие функции $f$ имеют вид

$$
\begin{aligned}
& f=a v e^{\sqrt{2} u}+b w e^{-\sqrt{2} u}, \\
& f=a v^{2} e^{2 u}+b w^{2} e^{-2 u}, \\
& f=a v^{2} e^{2 u}+b w e^{-u} \\
& f=a v e^{u}+b w e^{-u}
\end{aligned}
$$




$$
\begin{aligned}
& f=\left(v w+\frac{c}{2}\right)\left[a e^{\sqrt{2} u}+b e^{-\sqrt{2} u}\right], \\
& f=a\left(v w+\frac{c}{2}\right) e^{\sqrt{2} u}+b e^{-\sqrt{2} u}, \\
& f=a\left(v w+\frac{c}{2}\right) e^{\sqrt{2} u}+b e^{-2 \sqrt{2} u}, \\
& f=\left(v^{2} w+\frac{2}{3} v c\right) e^{\sqrt{2} u} \\
& f=v e^{-\sqrt{2 / 3} u}
\end{aligned}
$$

где $a$ и $b$ - произвольные постоянные.

В настоящей работе мы рассматриваем системы (12) с функциями (13a)-(13и) при условии $a b=0$. Две основные цели, которые мы преследуем здесь, - это доказательство лиувиллевости рассматриваемых систем и построение эволюционных систем, связанных дифференциальными подстановками с симметриями гиперболических систем (13a)-(13ж). Для доказательства лиувиллевости мы используем идею вычисления псевдоконстант, приведенную выше. Как будет показано, все полученные эволюционные системы обладают по крайней мере одной локальной гамильтоновой структурой. Случай $a b \neq 0$ будет рассмотрен в отдельной публикации, где будет показано, что системы (13a)-(13ж) имеют нетривиальные представления Лакса и, следовательно, интегрируемы методом обратной задачи рассеяния.

\section{2. СИСТЕМЫ ЛИУВИЛЛЕВСКОГО ТИПА}

Если положить $a=0$ или $b=0$ в системах (13a)-(13ж), то можно получить следуюшие четыре неэквивалентные системы:

$$
\begin{aligned}
& u_{x t}=\sqrt{2} a v e^{\sqrt{2} u}, \quad v_{x t}=\psi w v_{x} v_{t} \\
& w_{x t}=a \psi^{-1} e^{\sqrt{2} u}+\psi v w_{x} w_{t} \\
& u_{x t}=2 a v^{2} e^{2 u}, \quad v_{x t}=\psi w v_{x} v_{t} \\
& w_{x t}=2 a v \psi^{-1} e^{2 u}+\psi v w_{x} w_{t} \\
& u_{x t}=a v e^{u}, \quad v_{x t}=\psi w v_{x} v_{t} \\
& w_{x t}=a \psi^{-1} e^{u}+\psi v w_{x} w_{t} \\
& u_{x t}=\sqrt{2} a\left(v w+\frac{c}{2}\right) e^{\sqrt{2} u} \\
& v_{x t}=a v \psi^{-1} e^{\sqrt{2} u}+\psi w v_{x} v_{t} \\
& w_{x t}=a w \psi^{-1} e^{\sqrt{2} u}+\psi v w_{x} w_{t} .
\end{aligned}
$$

Этот список не включает в себя треугольные системы, получающиеся из (13д)-(13ж) и состояшие из уравнения Лиувилля и вырождения системы Лунда-Редже. Функциям 
(133), (13и) соответствуют системы

$$
\begin{aligned}
& u_{x t}=\sqrt{2} v\left(v w+\frac{2}{3} c\right) e^{\sqrt{2} u} \\
& v_{x t}=v^{2} e^{\sqrt{2} u} \psi^{-1}+v_{t} v_{x} w \psi \\
& w_{x t}=2\left(v w+\frac{c}{3}\right) e^{\sqrt{2} u} \psi^{-1}+w_{t} w_{x} v \psi ; \\
& u_{x t}=-v \sqrt{\frac{2}{3}} e^{-\sqrt{2 / 3} u}, \quad v_{x t}=v_{t} v_{x} \psi w, \\
& w_{x t}=e^{-\sqrt{2 / 3} u} \psi^{-1}+w_{t} w_{x} v \psi
\end{aligned}
$$

В силу инвариантности систем (14)-(19) относительно преобразования $x \leftrightarrow t$ достаточно знать набор, состояший из трех $t$-псевдоконстант, удовлетворяюших первому из условий (6). Поэтому в дальнейшем мы ограничимся рассмотрением фактов, связанных только с $t$-псевдоконстантами, подразумевая справедливость аналогичных утверждений для $x$-псевдоконстант. Отметим также, что все рассматриваемые системы соответствуют функции $f$ вида

$$
f=g(v, w) e^{k u},
$$

и, следовательно, допускают по крайней мере одну псевдоконстанту вида [11], [12]

$$
\rho=u_{x x}-k \psi v_{x} w_{x}-\frac{k}{2} u_{x}^{2}
$$

Этот результат можно обобшить для случая приводимого риманова пространства $\mathbb{V}_{m}$ с метрикой вида

$$
d s^{2}=d u^{1} d u^{1}+g_{i j}(u) d u^{i} d u^{j}, \quad i, j=2, \ldots, m
$$

где $g_{i j}$ не зависит от $u^{1}$, и пусть $\Gamma_{j l}^{i}$ - связность, согласованная с неприводимой частью метрики (21). Справедлива следуюшая

Теорема 1. Любая система вида

$$
u_{x t}^{1}=k h e^{k u^{1}}, \quad u_{x t}^{i}+\Gamma_{j l}^{i} u_{x}^{j} u_{t}^{l}=g^{i j} \frac{\partial h}{\partial u^{j}} e^{k u^{1}}, \quad i, j, l=2, \ldots, m,
$$

әде $k=$ const, $\quad h=h\left(u^{2}, \ldots, u^{m}\right)-$ произвольная функиия, допускает псевдоконстанту

$$
\rho=u_{x x}^{1}-\frac{k}{2}\left(\left(u_{x}^{1}\right)^{2}+g_{i j} u_{x}^{i} u_{x}^{j}\right)
$$

а векторное поле

$$
u_{\tau}^{1}=\left(k u_{x}^{1}+D\right) \rho, \quad u_{\tau}^{s}=k u_{x}^{s} \rho, \quad s=2, \ldots, m
$$


является высшей симметрией.

Справедливость теоремы можно проверить прямым вычислением. Отметим, что симметрия (22) приводится к треугольной системе

$$
\rho_{\tau}=\rho_{x x x}+3 k \rho \rho_{x}, \quad u_{\tau}^{i}=u_{x}^{i} \rho, \quad i>1,
$$

дифференциальной подстановкой $\left(u^{1}, u^{i}\right) \rightarrow\left(\rho, u^{i}\right), i>1$. Этот пример показывает, что далеко не во всех случаях действие оператора $D_{\tau}$ на псевдоконстанты приводит к новым псевдоконстантам. Симметрии, обладаюшие таким свойством, мы называем вырожденными; точное определение сформулируем следующим образом.

ОПРЕДЕЛЕНИЕ 2. Высшая симметрия

$$
u_{\tau}^{i}=\sigma^{i}\left(x, t, \mathbf{u}, \mathbf{u}_{1}, \ldots\right)
$$

гиперболической системы (12) называется вырожденной, если она может быть приведена к блочно-треугольному виду

$$
\begin{array}{ll}
\omega_{\tau}^{i}=\phi^{i}\left(\omega^{1}, \ldots, \omega^{k}\right), & i=1, \ldots, k, \\
\omega_{\tau}^{j}=\phi^{j}\left(\omega^{1}, \ldots, \omega^{m}\right), & j=k+1, \ldots, m,
\end{array}
$$

где $\phi^{i}, \phi^{j}$ - дифференциальные функции, с помошью некоторой дифференциальной подстановки

$$
\omega^{i}=\omega^{i}\left(x, \mathbf{u}, \mathbf{u}_{1}, \ldots\right), \quad i=1, \ldots, m
$$

Нужно признать, что приведенное определение не дает четкого алгоритма проверки того, является ли рассматриваемая симметрия вырожденной или нет. Поэтому интересно было бы отыскать сравнительно легко проверяемое условие, дающее ответ на этот вопрос. Следствием последнего определения и инвариантности $x \leftrightarrow t$ уравнения (12) является следующее

УТВЕРЖДЕНИЕ 1. Если система (12) допускает невырожденную высшую симметрию и одну псевдоконстанту, то она является системой лиувиллевского ти$n a$.

Приступим теперь к построению полных наборов псевдоконстант. Рассмотрим функцию вида

$$
f=a v^{l} e^{k u}
$$

где $a, k, l=\mathrm{const}, \quad k \neq 0$. Отметим, что функции вида (25) соответствуют системы (14)-(16), а также система (19).

ТЕОРема 2. Любая система вида (12), где $f$ имеет вид (25), допускает по крайней мере две псевдоконстанты $\rho, \theta$, где $\rho$ задается формулой (20), а $\theta$ имеет вид

$$
\theta=D \ln v_{x}+\frac{l}{k} u_{x}-w_{x} v \psi
$$


ДокАЗАТЕЛЬСТВо. Наличие псевдоконстанты $\rho$ следует из теоремы 1. Заметим, что в рассматриваемом случае второе из уравнений (12) можно представить в виде

$$
D_{t} \ln v_{x}=v_{t} w \psi
$$

Отсюда получаем

$$
D\left(D_{t} \ln v_{x}+\frac{l}{k} u_{t}\right)=D\left(v_{t} w \psi+\frac{l}{k} u_{t}\right) .
$$

Используя закон сохранения $D\left(v_{t} w \psi+(l / k) u_{t}\right)=D_{t}\left(w_{x} v \psi\right)$, получим

$$
D_{t}\left(D\left(\ln v_{x}\right)+\frac{l}{k} u_{x}-w_{x} v \psi\right)=0 .
$$

Таким образом, для систем (14)-(16) и (19) доказано наличие по крайней мере двух псевдоконстант.

Для отыскания третьей псевдоконстанты $\phi$ удобно использовать следуюший факт: все рассматриваемые системы допускают невырожденные симметрии. Подействовав оператором $D_{\sigma} \equiv D_{\tau}$ на функции $\rho$ или $\theta$, где $\sigma$ - симметрия, мы можем получить третью псевдоконстанту. Например, система (14) допускает следуюшую симметрию второго порядка [12]:

$$
\begin{gathered}
u_{\tau}=\sqrt{2} \psi v_{x} w_{x}, \quad v_{\tau}=v_{x x}-2 v \psi v_{x} w_{x}+\sqrt{2} u_{x} v_{x} \\
w_{\tau}=-w_{x x}+2 w \psi v_{x} w_{x}+\sqrt{2} u_{x} w_{x} .
\end{gathered}
$$

Положим $\hat{\phi}=D_{\sigma} \rho$, где $\rho$ имеет вид (20), причем $k=\sqrt{2}$. Тогда нетрудно проверить, что $\hat{\phi} \in \operatorname{Im} D$. Интегрируя $\hat{\phi}$ по $x$, получим

$$
\phi=D^{-1} \hat{\phi}=2 \sqrt{2} \psi v_{1}\left(w_{2}-v_{1} w_{1} \psi w-\sqrt{2} u_{1} w_{1}\right) .
$$

Это также дает нам первое уравнение системы, связанной дифференциальной подстановкой $(\rho, \theta, \phi)$ с системой (26). Таким образом, полный набор псевдоконстант для системы (14) можно взять в виде

$$
\begin{aligned}
\rho & =\frac{1}{6}\left(\sqrt{2} u_{2}-u_{1}^{2}-2 v_{1} w_{1} \psi\right), \\
\theta & =v_{2} v_{1}^{-1}+\frac{\sqrt{2}}{2} u_{1}-\psi w_{1} v, \\
\phi & =\psi v_{1}\left(w_{2}-v_{1} w_{1} \psi w-\sqrt{2} u_{1} w_{1}\right) .
\end{aligned}
$$


Так же просто получаются полные наборы псевдоконстант для систем (15), (16); их явный вид дают следуюшие формулы:

$$
\begin{aligned}
\rho= & u_{2}-u_{1}^{2}-2 v_{1} w_{1} \psi \\
\theta= & v_{2} v_{1}^{-1}+u_{1}-v \psi w_{1}, \\
\phi= & -3 \psi\left(2 v_{1}^{3} w_{1} w^{2} \psi^{2}-2 \psi w w_{2} v_{1}^{2}+\psi v w_{1} w_{2} v_{1}-2 \psi v v_{1} w_{1}^{2} u_{1}-\right. \\
& \quad-2 \psi v_{1}^{2} w_{1}^{2}-2 v_{1} w_{1} u_{2}+2 v_{2} u_{1} w_{1}-v_{2} w_{2}-4 w_{2} u_{1} v_{1}+4 v_{1} w_{1} u_{1}^{2}+ \\
& \left.+4 w \psi v_{1}^{2} w_{1} u_{1}+v_{1} w_{3}\right) ; \\
\rho= & 2 u_{2}-u_{1}^{2}-2 v_{1} w_{1} \psi \\
\theta= & v_{2} v_{1}^{-1}-w_{1} v \psi+u_{1}, \\
\phi= & v_{1} \psi\left(-w_{3}-2 w^{2} \psi^{2} w_{1} v_{1}^{2}-v w \psi^{2} v_{1} w_{1}^{2}+2 \psi w v_{1} w_{2}-3 \psi w v_{1} w_{1} u_{1}+\right. \\
& \left.+\frac{3}{2} \psi w_{1}^{2} v_{1}+w \psi v_{2} w_{1}+w_{1} u_{2}+3 u_{1} w_{2}-2 w_{1} u_{1}^{2}\right) .
\end{aligned}
$$

Для системы (17) в силу теоремы 1 первую псевдоконстанту можно взять в виде

$$
\rho=\sqrt{2} u_{2}-u_{1}^{2}-2 \psi v_{1} w_{1}
$$

Рассмотрим функцию $\hat{\theta}=D_{\sigma} \rho$, где $\sigma$ - одна из невырожденных высших симметрий системы (17). Пусть, например, $\sigma$ - симметрия третьего порядка:

$$
\begin{aligned}
u_{\tau} & =-4 u_{x} v_{x} w_{x} \psi+\sqrt{2}\left(w_{x} v_{x x}+v_{x} w_{x x}\right) \psi-\sqrt{2} v_{x} w_{x}\left(v_{x} w+w_{x} v\right) \psi^{2}, \\
v_{\tau} & =v_{x x x}-3 w_{x} v_{x x} v \psi-\sqrt{2} v_{x} u_{x x}-2 v_{x} u_{x}^{2}+3 \psi^{2} v_{x} w_{x}^{2} v^{2}-v_{x}^{2} w_{x} \psi, \\
w_{\tau} & =w_{x x x}-3 v_{x} w_{x x} w \psi-\sqrt{2} w_{x} u_{x x}-2 w_{x} u_{x}^{2}+3 \psi^{2} w^{2} v_{x}^{2} w_{x}-v_{x} w_{x}^{2} \psi
\end{aligned}
$$

Отметим, что система (31) является также симметрией системы (13ж). Нетрудно проверить, что $\hat{\theta} \in \operatorname{Im} D$. Полагая $\theta=D^{-1} \hat{\theta} / 3$, получим явный вид функции $\theta$ :

$$
\theta=g_{1} g_{2} \psi
$$

где

$$
\begin{aligned}
& g_{1}=2 v_{1} u_{1}+\sqrt{2} v \psi v_{1} w_{1}-\sqrt{2} v_{2}, \\
& g_{2}=2 w_{1} u_{1}+\sqrt{2} w \psi v_{1} w_{1}-\sqrt{2} w_{2} .
\end{aligned}
$$

Можно проверить, что функции $g_{1}, g_{2}$ удовлетворяют уравнениям

$$
D_{t} \ln g_{1}=v_{t} w \psi, \quad D_{t} \ln g_{2}=w_{t} v \psi \text {. }
$$

Используя закон сохранения системы (17)

$$
D\left(v_{t} w \psi\right)=D_{t}\left(w_{x} v \psi\right)
$$


нетрудно получить следствия:

$$
D_{t}\left(-w_{x} v \psi+D \ln g_{1}\right)=0, \quad D_{t}\left(-v_{x} w \psi+D \ln g_{2}\right)=0
$$

Следовательно, в качестве третьей независимой псевдоконстанты можно взять любую из функций

$$
\phi=\theta\left(-w_{x} v \psi+D \ln g_{1}\right), \quad \tilde{\phi}=\theta\left(-v_{x} w \psi+D \ln g_{2}\right) .
$$

Отметим, что $\phi, \tilde{\phi}$ связаны соотношением $\theta_{x}=\phi+\tilde{\phi}$.

Для системы (18) первая псевдоконстанта имеет вид

$$
\rho=\sqrt{2} u_{2}-u_{1}^{2}-2 \psi v_{1} w_{1}
$$

Пусть функция $g_{1}$ определяется формулой (33). Нетрудно проверить, что и в рассматриваемом случае $g_{1}$ удовлетворяет уравнению (34). Используя закон сохранения системы (18)

$$
D_{t}\left(w_{x} v \psi-\frac{\sqrt{2}}{2} u_{x}\right)=D\left(v_{t} w \psi\right)
$$

получим следствие

$$
D_{t}\left(D\left(\ln g_{1}\right)-w_{x} v \psi+\frac{\sqrt{2}}{2} u_{x}\right)=0
$$

откуда

$$
\theta=D\left(\ln g_{1}\right)-w_{x} v \psi+\frac{\sqrt{2}}{2} u_{x}
$$

Псевдоконстанту фможно получить с помошью какой-либо невырож денной высшей симметрии системы (18). В данном случае $\min \operatorname{ord}(\sigma)=5$ :

$$
u_{\tau}=-4 u_{5}+\cdots, \quad v_{\tau}=v_{5}+\cdots, \quad w_{\tau}=w_{5}+\cdots
$$

здесь мы не приводим полные выражения из-за их громоздкости. Положим

$$
\phi=D_{\sigma} \rho+4 \rho_{x x x x x}+20 \rho \rho_{x x x}
$$

Тогда можно проверить, что $\phi$ имеет вид

$$
\phi=-\frac{5}{\sqrt{2}} g_{1} w_{5} \psi+\phi_{1}
$$

где $\operatorname{ord}\left(\phi_{1}\right)=4$. Следовательно, набор $(\rho, \theta, \phi)$ является полным. Функцию $\phi_{1}$ мы не приводим из-за ее громоздкости.

Для системы (19) из теоремы 2 следует, что первые две псевдоконстанты имеют вид

$$
\begin{aligned}
& \rho=\sqrt{6} u_{2}+u_{1}^{2}+2 v_{1} w_{1} \psi, \\
& \theta=v_{2} v_{1}^{-1}-\frac{\sqrt{6}}{2} u_{1}-v w_{1} \psi
\end{aligned}
$$


Рассмотрим функцию

$$
\hat{\phi}=D_{\sigma} \rho+\frac{53}{2} \rho_{x x x x x}
$$

где $\sigma$ - симметрия пятого порядка системы (19):

$$
u_{\tau}=-\frac{53}{2} u_{5}+\cdots, \quad v_{\tau}=v_{5}+\cdots, \quad w_{\tau}=w_{5}+\cdots
$$

Можно проверить, что $\hat{\phi} \in \operatorname{Im} D$. Тогда, полагая $\phi=D^{-1} \hat{\phi}$, получим

$$
\phi=5 \psi v_{1} w_{5}+\phi_{1}
$$

причем ord $\left(\phi_{1}\right)=4$. Следовательно, функции $(\rho, \theta, \phi)$ представляют полный набор псевдоконстант. Функция $\phi_{1}$ довольно громоздкая, и мы ее здесь не приводим.

\section{3. ДИФФЕРЕНЦИАЛЬНЫЕ ПОДСТАНОВКИ ДЛЯ СИММЕТРИЙ СИСТЕМ ЛИУВИЛЛЕВСКОГО ТИПА}

Важной особенностью уравнений и систем лиувиллевского типа является их связь с преобразованиями типа Миуры. А именно, справедливо следуюшее (см. [2], [10], [13])

УТВЕРЖДЕНИЕ 2. Для любой симметрии (24) системы (5), допускающей полный набор псевдоконстант, существует әволючионная система

$$
\omega_{\tau}^{i}=\Phi^{i}\left(\boldsymbol{\omega}, \boldsymbol{\omega}_{x}, \ldots\right)
$$

связанная с ней дифференциальной подстановкой $\omega^{i}=\omega^{i}\left(\mathbf{u}, \mathbf{u}_{x}, \ldots\right), \quad i=1, \ldots, n$, где $D_{\tau}$ - оператор эволючионного дифференцирования в силу симметрии, $\omega^{i}$ псевдоконстанты.

Как уже было отмечено, операторы $D_{\tau}$ и $D_{t}$ коммутируют, и, таким образом, оператор $D_{\tau}$ переводит псевдоконстанты системы (5) снова в псевдоконстанты. Понятно, что для гиперболической системы (5) число независимых псевдоконстант по каждой характеристике не может быть больше $n$, поэтому выражение, стоящее в левой части (37), всегда можно выразить через псевдоконстанты $\omega^{i}$ и полные производные от них.

Среди симметрий систем (14)-(17) нас будут интересовать только те, которые одновременно являются симметриями для полных систем (13a)-(13ж). Такой выбор обусловлен тем, что эти системы являются интегрируемыми методом обратной задачи рассеяния.

Рассмотрим гиперболическую систему (5), и пусть функции $\omega^{i}\left(\mathbf{u}, \mathbf{u}_{1}, \ldots\right)$ представляют полный набор ее псевдоконстант. Предположим, что высшие симметрии системы (5) представимы в виде (ср. с формулой (2))

$$
u_{\tau}^{i}=M^{i j}\left(\mathbf{u}, \mathbf{u}_{1}, \ldots\right) f_{j}(\boldsymbol{\omega}),
$$


где $M^{i j}$ - матричный линейный дифференциальный оператор. Действуя оператором $D_{\tau}$ на $\omega^{i}$, будем иметь

$$
\omega_{\tau}^{i}=\frac{\partial \omega^{i}}{\partial u_{k}^{j}} D^{k} M^{j l} f_{l},
$$

или, в матричной форме,

$$
\boldsymbol{\omega}_{\tau}=\boldsymbol{\omega}^{\prime} M \mathbf{f}(\boldsymbol{\omega})=L \mathbf{f}(\boldsymbol{\omega})
$$

где штрих обозначает производную Фреше. В соответствии с утверждением 2 линейный оператор $L$ всегда выражается через псевдоконстанты системы (5); таким образом, эволюционная система (39), записанная в переменных $\omega^{i}$, и есть искомая система, связанная подстановкой $\boldsymbol{\omega}=\boldsymbol{\omega}\left(\mathbf{u}, \mathbf{u}_{1}, \ldots\right)$ с (38). Отметим, что для скалярного уравнения лиувиллевского типа представление любой симметрии в форме (38) всегда возможно. В случае систем такое представление можно найти, если системе соответствует завершающаяся нулем последовательность обобщенных инвариантов Лапласа [14]. Однако для систем, имеющих гамильтонову форму, существует более простой способ отыскания оператора $M$.

ТЕОРема 3. Пусть система лиувиллевского типа (5) имеет гамильтонову фор$\mathcal{M} y$

$$
u_{t}^{\alpha}=J^{\alpha \beta} \frac{\delta H(u)}{\delta u^{\beta}},
$$

где $J$ - гамильтонов оператор, $H$ - соответствующий гамильтониан. Тогда следующая эволюиионная система является симметрией системь (41) при произвольной функиии $f=f\left(\boldsymbol{\omega}, \boldsymbol{\omega}_{x}, \ldots\right)$ :

$$
u_{\tau}^{\alpha}=\sum_{\beta, \gamma} J^{\alpha \beta}\left[\left(\omega^{\beta}\right)_{\gamma}^{\prime}\right]^{+} \frac{\delta f}{\delta \omega^{\gamma}},
$$

где “+” означает формальное сопряжение.

ДокАЗАТЕЛЬство. Из равенства $D_{t} \omega^{\alpha}=0$ вытекает соотношение

$$
\left(\omega^{\alpha}\right)_{\beta}^{\prime} J^{\beta \gamma} \frac{\delta H}{\delta u^{\gamma}}=0
$$

Вычислим значение скобки Пуассона, порождаемой оператором $J$, для произвольной функции $f$ :

$$
\begin{aligned}
\{H, f\} & =\int \frac{\delta H}{\delta u^{\alpha}} J^{\alpha \beta} \frac{\delta f}{\delta u^{\beta}} d x=\int \frac{\delta H}{\delta u^{\alpha}} \sum_{\beta, \gamma} J^{\alpha \beta}\left[\left(\omega^{\beta}\right)_{\gamma}^{\prime}\right]^{+} \frac{\delta f}{\delta \omega^{\gamma}} d x= \\
& =-\int \frac{\delta H}{\delta u^{\alpha}}\left[\left(\omega^{\alpha}\right)_{\beta}^{\prime} J^{\gamma \beta}\right]^{+} \frac{\delta f}{\delta \omega^{\gamma}} d x=-\int \frac{\delta f}{\delta \omega^{\alpha}}\left(\omega^{\alpha}\right)_{\beta}^{\prime} J^{\gamma \beta} \frac{\delta H}{\delta u^{\gamma}} d x=0 .
\end{aligned}
$$

Отсюда следует, что эволюционная система

$$
u_{\tau}^{\alpha}=J^{\alpha \beta} \frac{\delta f}{\delta u^{\beta}}=\sum_{\gamma, \beta} J^{\alpha \beta}\left[\left(\omega^{\beta}\right)_{\gamma}^{\prime}\right]^{+}\left(\frac{\delta f}{\delta \omega^{\gamma}}\right)
$$

является симметрией для (41). 
ЗАмЕЧАНИЕ 1. Отметим, что под знаком последнего интеграла в формуле (43) можно сделать замену

$$
\frac{\delta f}{\delta \omega^{\alpha}} \rightarrow f_{\alpha}\left(\boldsymbol{\omega}, \boldsymbol{\omega}_{x}, \ldots\right)
$$

где $f_{\alpha}-$ произвольные функции. Следовательно, эволюционная система

$$
u_{\tau}^{\alpha}=\sum_{\beta, \gamma} J^{\alpha \beta}\left[\left(\omega^{\beta}\right)_{\gamma}^{\prime}\right]^{+} f_{\gamma}
$$

также будет симметрией системы (41).

ЗАмЕчАниЕ 2. Система (45) имеет форму (38), так что оператор

$$
M=J \omega^{\prime+}
$$

отображает псевдоконстанты в симметрии системы (41). Таким же свойством будет обладать любой оператор вида

$$
\widehat{M}=M S
$$

где $S=S\left(\boldsymbol{\omega}, \boldsymbol{\omega}_{x}, \ldots\right)$ - некоторая матрица. Однако в дальнейшем мы будем иметь дело только с операторами, имеюшими форму (46). Далее, из закона преобразования гамильтонова оператора при преобразовании $\boldsymbol{\omega}=\boldsymbol{\omega}\left(\mathbf{u}, \mathbf{u}_{1}, \ldots\right)$ следует, что $L$ представляет гамильтонов оператор для системы (39); действительно,

$$
\tilde{J}=\omega^{\prime} J \omega^{\prime+}=\omega^{\prime} M=L
$$

ЗАмЕчАнИЕ 3 . Особый интерес представляет случай, когда эволюционные системы (38), (39) являются интегрируемыми методом обратной задачи рассеяния. Пусть система (39) гамильтонова с оператором $\boldsymbol{\omega}^{\prime} M$ и имеет бесконечную последовательность канонических плотностей

$$
\frac{\delta f_{i}}{\delta \omega}=\widehat{R}^{+} \frac{\delta f_{i-1}}{\delta \boldsymbol{\omega}}
$$

где $\widehat{R}^{+}$- градиентно-рекурсионный оператор, и пусть $R$ - симметрийно-рекурсионный оператор для соответствуюшей иерархии системы (38). Тогда мы можем записать

$$
\boldsymbol{\omega}^{\prime} R M \frac{\delta f_{i-1}}{\delta \boldsymbol{\omega}}=\boldsymbol{\omega}^{\prime} M \frac{\delta f_{i}}{\delta \boldsymbol{\omega}}=\boldsymbol{\omega}^{\prime} M \widehat{R}^{+} \frac{\delta f_{i-1}}{\delta \boldsymbol{\omega}}
$$

откуда следует, что

$$
R=M \widehat{R}^{+} M^{-1}
$$


ПримеР. Система (7) имеет гамильтонову форму, причем

$$
J=\left(\begin{array}{ll}
2 & 1 \\
1 & 2
\end{array}\right) D^{-1}, \quad H=\frac{1}{3}\left(e^{2 u-v}+e^{-u+2 v}\right) .
$$

Подставляя в формулу (46) выражение для оператора $J$ и $\omega=(\rho, \theta)$, получим явный вид оператора $M$ :

$$
M=\left(\begin{array}{cc}
3 u_{x}+3 D & 2 u_{x} v_{x}-2 v_{x}^{2}+2 v_{x x}-u_{x x}+u_{x}^{2}-D^{2} \\
3 v_{x}+3 D & 2 u_{x}^{2}-2 u_{x} v_{x}-v_{x}^{2}+v_{x x}-2 u_{x x}-3 v_{x} D-2 D^{2}
\end{array}\right) .
$$

Систему (8) можно записать в виде

$$
\left(\begin{array}{l}
u_{\tau} \\
v_{\tau}
\end{array}\right)=M\left(\begin{array}{c}
0 \\
-1
\end{array}\right)
$$

Далее, формула (47) дает

$$
L=3\left(\begin{array}{cc}
2 D^{3}+\rho D+D \rho & -D^{4}-\rho D^{2}+\alpha D-\rho_{x x}+2 \theta_{x} \\
D^{4}+D^{2} \rho+D \alpha+\rho_{x x}-2 \theta_{x} & \frac{-2 D^{5}-2\left(D^{3} \rho+\rho D^{3}\right)+2 \beta D+\beta_{x}}{3}
\end{array}\right),
$$

где

$$
\alpha=3 \theta-2 \rho_{x}, \quad \beta=3 \theta_{x}-\rho^{2} .
$$

Подействовав оператором $L$ на $(0,-1)^{\mathrm{T}}$, получим систему $(9),(10)$. Эта система бигамильтонова (см., например, [15]), а первый гамильтонов оператор имеет вид

$$
L_{1}=\left(\begin{array}{cc}
0 & D \\
D & 0
\end{array}\right)
$$

Перейдем теперь к построению эволюционных систем, связанных дифференциальными подстановками (27)-(29), (30), (32), (35) с симметриями систем (13a)-(13ж).

1. Система (14). Полный набор псевдоконстант $\left(\omega^{1}, \omega^{2}, \omega^{3}\right)=(\rho, \theta, \phi)$ дан формулами (27). Как показано в работах [12], система (12) является гамильтоновой с оператором

$$
J=\left(\begin{array}{ccc}
D^{-1} & 0 & 0 \\
0 & 0 & e^{\varphi} D^{-1} e^{-\varphi} \psi^{-1} \\
0 & \psi^{-1} e^{-\varphi} D^{-1} e^{\varphi} & 0
\end{array}\right)
$$

где $\varphi=D^{-1}\left(\psi v_{x} w\right)$. Подставляя операторы $J, \omega^{\prime+}$ в формулу (46), получим

$$
M=\left(\begin{array}{ccc}
\frac{1}{3} u_{x}+\frac{\sqrt{2}}{6} D & -\frac{\sqrt{2}}{2} & \sqrt{2} \psi v_{x} w_{x} \\
\frac{1}{3} v_{x} & v & v_{x x}-v_{x}\left(2 v \psi w_{x}-\sqrt{2} u_{x}-D\right) \\
\frac{1}{3} w_{x} & \frac{1}{\psi v_{x}} D-w & -w_{x x}+2 w \psi v_{x} w_{x}+\sqrt{2} u_{x} w_{x}
\end{array}\right)
$$

Симметрии второго и третьего порядка системы (13a) имеют соответственно вид

$$
\mathbf{u}_{\tau}=M(0,0,1)^{\mathrm{T}}, \quad \mathbf{u}_{\tau}=M\left(\frac{9}{2} \rho, \phi, \theta\right)^{\mathrm{T}} .
$$


Отметим, что первая система в (51) есть не что иное, как система (26). Оператор $L$ имеет вид

$$
L=\frac{1}{3}\left(\begin{array}{ccc}
\frac{1}{6} D^{3}+2 \rho D+\rho_{x} & -\frac{3}{2} D^{2}+\theta D & 3 \phi D+2 \phi_{x} \\
\frac{3}{2} D^{2}+D \theta & \frac{9}{2} D & 3 D^{3}+6 D^{2} \theta+3 D \alpha \\
3 \phi D+\phi_{x} & 3 D^{3}-6 \theta D^{2}+3 \alpha D & 6 \beta D+3 \beta_{x}
\end{array}\right)
$$

где

$$
\alpha=3 \rho+\theta^{2}-\theta_{x}, \quad \beta=2 \theta \phi-\phi_{x}
$$

Эволюционные системы

$$
\boldsymbol{\omega}_{\tau}=L(0,0,1)^{\mathrm{T}}, \quad \boldsymbol{\omega}_{\tau}=L\left(\frac{9}{2} \rho, \phi, \theta\right)^{\mathrm{T}}
$$

связаны дифференциальной подстановкой (27) с эволюционными системами (51). Явный вид систем (53) таков:

$$
\begin{aligned}
\rho_{\tau} & =\frac{2}{3} \phi_{x} \\
\theta_{\tau} & =\theta_{x x}+\left(\theta^{2}\right)_{x}+3 \rho_{x} \\
\phi_{\tau} & =-\phi_{x x}+2(\theta \phi)_{x} \\
\rho_{\tau} & =\left(\frac{1}{4} \rho_{x x}-\frac{1}{2} \phi_{x}+\frac{9}{4} \rho^{2}+\theta \phi\right)_{x} \\
\theta_{\tau} & =\left(\theta_{x x}+\frac{9}{4} \rho_{x}+\frac{3}{2}\left(\theta^{2}\right)_{x}+\frac{9}{2} \rho \theta+\theta^{3}+\frac{3}{2} \phi\right)_{x}, \\
\phi_{\tau} & =\left(\phi_{x x}-3 \theta \phi_{x}+\frac{9}{2} \rho \phi+3 \phi \theta^{2}\right)_{x}
\end{aligned}
$$

Нетрудно найти, что системы (54), (55) имеют также следующий гамильтонов оператор первого порядка:

$$
L_{1}=\left(\begin{array}{ccc}
\frac{2}{9} D & 0 & 0 \\
0 & 0 & D \\
0 & D & 0
\end{array}\right)
$$

Операторы $L_{1}, L$ являются согласованными, поэтому $R=L L_{1}^{-1}$ - оператор рекурсии для систем (54), (55):

$$
R=\left(\begin{array}{ccc}
\frac{1}{4} D^{2}+3 \rho+\frac{3}{2} \rho_{x} D^{-1} & \phi+\frac{2}{3} \phi_{x} D^{-1} & \frac{1}{3} \theta-\frac{1}{2} D \\
\frac{9}{4} D+\frac{3}{2} D \theta D^{-1} & D^{2}+2 D^{2} \theta D^{-1}+D \alpha D^{-1} & \frac{3}{2} \\
\frac{9}{2} \phi+\frac{3}{2} \phi_{x} D^{-1} & 2 D \beta D^{-1}-\beta_{x} D^{-1} & D^{2}-2 \theta D+\alpha
\end{array}\right)
$$

Подействовав оператором $R$ на правые части систем (54), (55), мы получим две серии высших симметрий:

$$
\sigma_{2 k+2}=R^{k} \sigma_{0}, \quad \sigma_{2 k+3}=R^{k} \sigma_{1}
$$


Очевидно, что системы (54), (55) имеют высшие симметрии всех порядков, причем $\sigma_{2 k+2}-$ симметрия четного порядка, а $\sigma_{2 k+3}-$ нечетного. Отметим еще, что все системы этих иерархий имеют вид законов сохранения.

2. Cистема (15). Оператор $M$ имеет вид

$$
M=\left(\begin{array}{ccc}
2 u_{x}+D & -1 & 6 v_{x} \psi\left(w_{x} D+2 w_{x} \theta+\beta\right) \\
2 v_{x} & v & 3 v_{x}\left(D^{2}+4 \alpha D+\gamma\right)+v_{x x}(9 D+6 \alpha) \\
2 w_{x} & \frac{1}{\psi v_{x}} D-w & 3\left(w_{x x}-2 u_{x} w_{x}\right)(D+2 \theta)-\frac{2}{v_{x} \psi} \phi+6 w \psi v_{x} \beta
\end{array}\right)
$$

где

$$
\begin{gathered}
\alpha=u_{x}-v \psi w_{x}, \quad \beta=w \psi v_{x} w_{x}-w_{x x}+2 u_{x} w_{x} \\
\gamma=2\left(\theta_{x}+\theta^{2}+\alpha^{2}-\psi\left(v_{x} w_{x}+v \beta\right)\right) .
\end{gathered}
$$

Симметрии третьего порядка систем (13б), (13в) имеют соответственно вид

$$
\mathbf{u}_{\tau}=M\left(\frac{1}{4} \rho, 0, \frac{1}{6}\right)^{\mathrm{T}}, \quad \mathbf{u}_{\tau}=\frac{1}{6} M(0,0,1)^{\mathrm{T}}
$$

Оператор $L$ имеет вид

$$
L=\left(\begin{array}{ccc}
D^{3}+4 \rho D+2 \rho_{x} & -3 D^{2}+2 \theta D & 8 \phi D+6 \phi_{x} \\
3 D^{2}+2 D \theta & D & 3 D^{4}+12 D^{3} \theta-D^{2} \zeta+D \xi \\
8 D \phi-6 \phi_{x} & -3 D^{4}+12 \theta D^{3}+\zeta D^{2}+\xi D & 9\left(\phi D^{3}+D^{3} \phi\right)+\eta D+\frac{1}{2} \eta_{x}
\end{array}\right),
$$

где

$$
\begin{gathered}
\zeta=3\left(3 \theta_{x}-\rho-5 \theta^{2}\right), \quad \xi=6\left(\frac{1}{2} \theta_{x x}-\frac{1}{2} \rho_{x}-\left(\theta^{2}\right)_{x}+\theta^{3}+\rho \theta\right) \\
\eta=-6 \phi_{x x}-36 \phi_{x} \theta+12 \phi\left(\rho+3 \theta^{2}+3 \theta_{x}\right) .
\end{gathered}
$$

Набор псевдоконстант (28) задает дифференциальную подстановку систем (59) соответственно в системы

$$
\begin{aligned}
\rho_{\tau} & =\left(\frac{1}{4} \rho_{x x}+\frac{3}{4} \rho^{2}+\phi\right)_{x}, \\
\theta_{\tau} & =\left(\theta_{x x}+\frac{3}{2}\left(\left(\theta^{2}\right)_{x}+\theta \rho\right)+\theta^{3}+\frac{3}{4} \rho_{x}\right)_{x}, \\
\phi_{\tau} & =\left(\phi_{x x}+3\left(\phi \theta_{x}-\theta \phi_{x}+\phi \theta^{2}+\phi \rho\right)\right)_{x}-\frac{3}{2} \phi_{x} \rho ; \\
\rho_{\tau} & =\phi_{x} \\
\theta_{\tau} & =\left(\theta_{x x}+3 \theta \theta_{x}+\theta \rho+\theta^{3}\right)_{x}, \\
\phi_{\tau} & =\left(\phi_{x x}+3\left(\phi \theta_{x}-\theta \phi_{x}+\phi \theta^{2}\right)+\rho \phi\right)_{x} .
\end{aligned}
$$


3. Система (16). Оператор $M$ имеет вид

$$
M=\left(\begin{array}{ccc}
2\left(u_{x}+D\right) & -1 & D \psi v_{x} w_{x}+\psi v_{x}\left(3 \beta+4 u_{x} w_{x}\right) \\
2 v_{x} & v & D^{2} v_{x}-3 v_{x}\left(v \psi w_{x}-u_{x}\right)(D+\theta)+v_{x} \rho \\
2 w_{x} & \frac{1}{\psi v_{x}} D-w & D \alpha-\frac{\phi}{v_{x} \psi}+\frac{1}{2} \psi v_{x}\left(w\left(4 \beta+6 w_{x} u_{x}\right)-3 w_{x}^{2}\right)
\end{array}\right)
$$

где

$$
\alpha=w \psi v_{x} w_{x}, \quad \beta=\alpha-w_{x x} .
$$

Система (13г) допускает следуюшую симмметрию третьего порядка:

$$
\mathbf{u}_{\tau}=M\left(-\frac{1}{8} \rho, 0,1\right)^{\mathrm{T}}
$$

Оператор $L$ имеет вид

$$
L=\left(\begin{array}{ccc}
4 D^{3}+4 D \rho-2 \rho_{x} & -4 D^{2}+2 \theta D & 8 \phi D+6 \phi_{x} \\
4 D^{2}+2 D \theta & D & D^{4}+3 D^{3} \theta-D^{2} \zeta+D \xi \\
8 D \phi-6 \phi_{x} & -D^{4}+3 \theta D^{3}+\zeta D^{2}+\xi D & \phi D^{3}+D^{3} \phi+2 \eta D+\eta_{x}
\end{array}\right),
$$

где

$$
\zeta=3 \theta_{x}-\rho-3 \theta^{2}, \quad \xi=\frac{1}{2} \zeta_{x}-\frac{1}{2} \theta_{x x}+\theta^{3}+\rho \theta, \quad \eta=\phi\left(\rho+3 \theta^{2}\right)-3 \phi_{x} \theta .
$$

Набор псевдоконстант (29) задает дифференциальную подстановку системы (64) в систему

$$
\begin{aligned}
\rho_{\tau} & =\left(-\frac{1}{2} \rho_{x x}+6 \phi-\frac{3}{8} \rho^{2}\right)_{x}, \\
\theta_{\tau} & =\left(\theta_{x x}+3 \theta \theta_{x}+\frac{3}{4} \theta \rho+\theta^{3}\right)_{x} \\
\phi_{\tau} & =\left(\phi_{x x}-3 \theta \phi_{x}+3 \phi \theta^{2}\right)_{x}+\frac{3}{4} \rho \phi_{x} .
\end{aligned}
$$

Кроме того, делая замену $u_{i} \rightarrow-u_{i}, v_{i} \rightarrow w_{i}, w_{i} \rightarrow v_{i}$ в (29), получим подстановку, которая связывает симметрию системы (13в) со следуюшей системой:

$$
\begin{aligned}
\rho_{\tau} & =6 \phi_{x}, \\
\theta_{\tau} & =\left(\theta_{x x}+3 \theta \theta_{x}+\theta \rho+\theta^{3}+\frac{1}{2} \rho_{x}\right)_{x}, \\
\phi_{\tau} & =\left(\phi_{x x}-3 \theta \phi_{x}+\rho \phi+3 \phi \theta^{2}\right)_{x} .
\end{aligned}
$$

4. Система (17). Оператор $M$ имеет вид

$$
M=\left(\begin{array}{lll}
M_{11} & M_{12} & M_{13} \\
M_{21} & M_{22} & M_{23} \\
M_{31} & M_{32} & M_{33}
\end{array}\right)
$$


где

$$
\begin{aligned}
M_{11}= & \sqrt{2} D+2 u_{x}, \quad M_{12}=-2 \psi\left(w_{x} g_{1}+v_{x} g_{2}\right) \\
M_{13}= & 2 \psi v_{x} g_{2} D+2 \psi\left(v_{x}\left(g_{2}\right)_{x}-w_{x}\left(g_{1}\right)_{x}\right)+2 \psi^{2}\left(v w_{x}^{2} g_{1}-w v_{x}^{2} g_{2}\right) \\
M_{21}= & 2 v_{x}, \quad M_{22}=-\sqrt{2} g_{1} D-\sqrt{2}\left(g_{1}\right)_{x}-2\left(u_{x}-\sqrt{2} \psi v w_{x}\right) g_{1}-\sqrt{2} v \psi v_{x} g_{2}, \\
M_{23}= & \sqrt{2}\left(v \psi\left(w_{x} g_{1}+v_{x} g_{2}\right)-\left(g_{1}\right)_{x}\right) D-\sqrt{2}\left(g_{1}\right)_{x x}+\sqrt{2}\left(3 v w_{x} \psi-\sqrt{2} u_{x}\right)\left(g_{1}\right)_{x}+ \\
& +\sqrt{2} \psi v v_{x}\left(g_{2}\right)_{x}-\sqrt{2}\left(3 \psi v^{2} w_{x}-v_{x}-2 \sqrt{2} v u_{x}\right) \psi w_{x} g_{1}-\sqrt{2} \psi^{2} v w v_{x}^{2} g_{2}, \\
M_{31}= & 2 w_{x}, \quad M_{32}=-\sqrt{2} g_{2} D+2 w_{x x}+2 \psi v_{x}\left(3 \psi w^{2} w_{x} v_{x}-w_{x}^{2}-3 w_{x x} w\right)- \\
& -2 w_{x}\left(2 u_{x}^{2}+\sqrt{2} u_{x x}\right), \\
M_{33}= & \sqrt{2} D^{2} g_{2}-\sqrt{2}\left(3 w \psi v_{x}-\sqrt{2} u_{x}\right) D g_{2}-\sqrt{2} w\left(w_{x} \psi\left(g_{1}\right)_{x}-w_{x}^{2} \psi^{2} v g_{1}\right)- \\
& -\sqrt{2} g_{2}\left(w_{x}-3 \psi w^{2} v_{x}+2 \sqrt{2} w u_{x}\right) \psi v_{x},
\end{aligned}
$$

а функции $g_{1}, g_{2}$ определяются по формулам (33). Систему (31) можно записать в виде

$$
\mathbf{u}_{\tau}=\frac{1}{2} M(0,1,0)^{\mathrm{T}}
$$

а симметрии третьего порядка систем (13д), (13е) соответственно как

$$
\mathbf{u}_{\tau}=\frac{1}{2} M(\rho, 1,0)^{\mathrm{T}}, \quad \mathbf{u}_{\tau}=\frac{1}{2} M\left(-\frac{1}{2} \rho, 1,0\right)^{\mathrm{T}} .
$$

Вид оператора $L$ таков:

где

$$
L=\left(\begin{array}{lll}
L_{11} & L_{12} & L_{13} \\
L_{21} & L_{22} & L_{23} \\
L_{31} & L_{32} & L_{33}
\end{array}\right)
$$

$$
\begin{aligned}
L_{11}= & 2 D^{3}+4 \rho D+2 \rho_{x}, \quad L_{12}=8 \theta D+6 \theta_{x}, \quad L_{13}=-4 D^{2} \theta+10 D \phi-2 \phi_{x}, \\
L_{21}= & 8 \theta D+2 \theta_{x}, \quad L_{22}=2\left(\theta D^{3}+D^{3} \theta\right)+4 \alpha D+2 \alpha_{x}, \\
L_{23}= & -2 D^{4} \theta+10 D^{3} \phi-2 D^{2}\left(6 \phi_{x}+\alpha\right)+D \gamma-\beta, \\
L_{31}= & 4 \theta D^{2}+10 \phi D+2 \phi_{x}, \quad L_{32}=2 \theta D^{4}+10 \phi D^{3}+2\left(6 \phi_{x}+\alpha\right) D^{2}+\gamma D+\beta, \\
L_{33}= & \left(4 \phi_{x}-\frac{8 \phi\left(\theta_{x}-\phi\right)}{\theta}\right) D^{3}+D^{3}\left(4 \phi_{x}-\frac{8 \phi\left(\theta_{x}-\phi\right)}{\theta}\right)+D \nu+\nu D, \\
\alpha= & 2 \rho \theta-\frac{3 \phi\left(\theta_{x}-\phi\right)}{\theta}, \\
\beta= & 2 \phi_{x x x}+8 \rho_{x} \phi+2 \theta \rho_{x x}+4 \phi_{x} \rho-\frac{6\left(\left(\theta_{x}-\phi\right) \phi_{x}\right)_{x}}{\theta}+ \\
& +6\left(\theta_{x}-\phi\right) \frac{\left(2 \theta_{x} \phi-(3 / 2) \phi^{2}\right)_{x}-\frac{12 \theta_{x} \phi\left(\theta_{x}-\phi\right)^{2}}{\theta^{3}}}{\theta^{2}} \\
\gamma= & 8 \phi_{x x}+6 \theta \rho_{x}+12 \rho \phi+\frac{2 \phi\left(8 \theta_{x}-7 \phi\right)\left(\theta_{x}-\phi\right)}{\theta^{2}}-\frac{2\left(\theta_{x x} \phi+8 \phi_{x} \theta_{x}-9 \phi \phi_{x}\right)}{\theta}, \\
\nu= & \theta^{2}-2 \phi_{x x x}+2 \rho_{x} \phi-\frac{4\left(2 \rho \phi\left(\theta_{x}-\phi\right)-3 \phi_{x}\left(\theta_{x}-\phi\right)_{x}-\phi\left(\theta_{x}-\phi\right)_{x x}\right)}{\theta}- \\
& \quad-\frac{4 \phi\left(3 \theta_{x x} \theta_{x}-3 \phi \phi_{x}+\phi \theta_{x} \theta_{x}-\theta_{x x} \phi\right)}{\theta^{2}}+\frac{8 \phi^{2}\left(2 \theta_{x}-\phi\right)\left(\theta_{x}-\phi\right)}{\theta^{3}}+4 \phi_{x} \rho .
\end{aligned}
$$

3 Теоретическая и математическая физика, т. 141, № 2, 2004 г. 
Полный набор псевдоконстант системы (17) задает дифференциальную подстановку систем (70), (71) в следуюшие системы, соответственно:

$$
\begin{aligned}
\rho_{\tau} & =3 \theta_{x} \\
\theta_{\tau} & =\left(\theta_{x x}-3 \phi \theta^{-1}\left(\theta_{x}-\phi\right)+2 \rho \theta\right)_{x}, \\
\phi_{\tau} & =\left(\phi_{x x}-3 \theta^{-2}\left(\theta_{x}-\phi\right)\left(\theta \phi_{x}-\phi \theta_{x}+\phi^{2}\right)+2 \phi \rho\right)_{x}+2 \rho_{x} \phi+\rho_{x x} \theta ; \\
\rho_{\tau} & =\left(\rho_{x x}+\frac{3}{2} \rho^{2}+3 \theta\right)_{x} \\
\theta_{\tau} & =\left(\theta_{x x}-3 \phi \theta^{-1}\left(\theta_{x}-\phi\right)+3 \theta\right)_{x}+3 \rho_{x} \theta, \\
\phi_{\tau} & =\left(\phi_{x x}-3 \theta^{-2}\left(\theta_{x}-\phi\right)\left(\theta \phi_{x}-\phi \theta_{x}+\phi^{2}\right)+3 \phi \rho\right)_{x}+6 \rho_{x} \phi+3 \rho_{x x} \theta ; \\
\rho_{\tau} & =\left(-\frac{1}{2} \rho_{x x}-\frac{3}{4} \rho^{2}+3 \theta\right)_{x}, \\
\theta_{\tau} & =\left(\theta_{x x}-3 \phi \theta^{-1}\left(\theta_{x}-\phi\right)\right)_{x}+\frac{3}{2} \rho \theta_{x}, \\
\phi_{\tau} & =\left(\phi_{x x}-3 \theta^{-2}\left(\theta_{x}-\phi\right)\left(\phi_{x} \theta-\theta_{x} \phi+\phi^{2}\right)+\frac{3}{2} \phi \rho\right)_{x} .
\end{aligned}
$$

\section{4. ЗАКЛЮЧЕНИЕ}

В данной работе доказано, что вырождения систем, найденных с помощью симметрийного подхода в работе [11], являются системами лиувиллевского типа. Также найдены новые эволюционные системы, связанные дифференциальными подстановками с симметриями исходных гиперболических систем. Интегрируемость полученных систем не вызывает сомнения; например, системы (54), (55) имеют рекурсионный оператор (57). Доказательство интегрируемости путем построения представлений Лакса или рекурсионных операторов для остальных систем мы надеемся вьполнить в одной из следующих публикаций.

\section{ПРИЛОЖЕНИЕ}

Как сообшил нам М. В. Павлов, эволюционная система (54) может быть связана с известной системой Яджимы-Оикавы [16]

$$
i \psi_{t}+\psi_{x x}-3 \rho \psi=0, \quad \rho_{t}=\frac{2}{3} \partial_{x}|\psi|^{2}
$$

следуюшим образом. Введем замену

$$
\psi=\sqrt{\phi} \exp \left(i \int \xi d x\right)
$$

В новых переменных система (77) примет вид

$$
\rho_{t}=\frac{2}{3} \phi_{x}, \quad \phi_{t}=-2 \partial_{x}(\phi \xi), \quad \xi_{t}=\partial_{x}\left(\frac{1}{2} \frac{\phi_{x x}}{\phi}-\frac{1}{4} \frac{\phi_{x}^{2}}{\phi^{2}}-\xi^{2}-3 \rho\right) .
$$


Делая подстановку $\xi=-\theta-(i / 2) \partial_{x} \ln (\phi)$ в $(78)$, получим

$$
\rho_{t}=\frac{2}{3} \phi_{x}, \quad \theta_{t}=-i \theta_{x x}+\left(\theta^{2}\right)_{x}+3 \rho_{x}, \quad \phi_{t}=i \phi_{x x}+2(\phi \theta)_{x}
$$

И, наконец, заменой $\partial_{t} \rightarrow i \partial_{t}, \partial_{x} \rightarrow i \partial_{x}$ система (79) приводится к виду (54).

Благодарности. Автор благодарен А. Г. Мешкову, М. В. Павлову и В. В. Соколову за полезные обсуждения.

\section{Список литературы}

[1] А. В. Жибер, А. Б. IIaбат. ДАН СССР. 1979. Т. 247. № 5. С. 1103-1107; R. K. Dodd, R. K. Bullough. Proc. Roy. Soc. London A. 1976. V. 351. P. 499-523.

[2] А. В. Жибер, В. В. Соколов. УМН. 2001. Т. 56. № 1. С. 63-106.

[3] А.Н. Лезнов, М. В. Савельев. Групповые методы интегрирования нелинейных динамических систем. М.: Наука, 1985.

[4] I. M. Anderson, N. Kamran. Duke Math. J. 1997. V. 87. № 2. P. 265-319; M. Juras, I. M. Anderson. Duke Math. J. 1997. V. 89. № 2. P. 351-375.

[5] E. Goursat. Lecons sur l'intégration des équations aux dérivées partielles du second ordre à deux variables indépendants. T. I, II. Paris: Hermann, 1896, 1898.

[6] А. В. Жибер. Изв. РАН. Сер. матем. 1994. Т. 58. № 4. С. 33-54.

[7] А. В. Жибер, В.В. Соколов. ТМФ. 1999. Т. 120. № 1. С. 20-26.

[8] А. Б. Шабат, Р. И. Ямилов. Экспоненциальные системы типа І и матрицы Картана. Препринт. Уфа: БФАН СССР, 1981.

[9] А. Н. Лезнов, В. Г. Смирнов, А. Б. Шабат. ТМФ. 1982. Т. 51. № 1. С. 10-22.

[10] В. В. Соколов. УМН. 1988. Т. 43. № 5. С. 133-163.

[11] D. K. Demskoi, A. G. Meshkov. New integrable string-like fields in $1+1$ dimensions. In: Proc. Second Int. Conf. Quantum Field Theory and Gravity (July 28 - August 2, 1997, Tomsk, Russia). Eds. I. L. Bukhbinder, K. E. Osetrin. Tomsk: Tomsk Pedagogical University, 1998. P. 282-285.

[12] Д. К. Демской, А.Г. Мешков. ТМФ. 2003. Т. 134. № 3. С. 401-415; D. K. Demskoi, A. G. Meshkov. Inverse Problems. 2003. V. 19. P. 563-571.

[13] C. Я. Стариев. ТМФ. 1998. Т. 116. № 3. С. 336-348.

[14] А. В. Жибер, С. Я. Старцев. Матем. заметки. 2003. Т. 74. № 6. С. 849-858.

[15] П. Олвер. Приложения групп Ли к дифференциальным уравнениям. М.: Мир, 1989.

[16] N. Yajima, M. Oikawa. Progr. Theor. Phys. 1976. V. 56. P. 1719-1739.

Поступила в редакцию 13.Х.2003 г., после доработки 16.III.2004 г. 